Research Article

\title{
Facile Synthesis of Curcumin-Loaded Starch-Maleate Nanoparticles
}

\author{
Suh Cem Pang, Soon Hiang Tay, and Suk Fun Chin \\ Department of Chemistry, Faculty of Resource Science and Technology, Universiti Malaysia Sarawak, 94300 Kota Samarahan, \\ Sarawak, Malaysia \\ Correspondence should be addressed to Suh Cem Pang; suhcem@gmail.com
}

Received 3 September 2013; Revised 18 December 2013; Accepted 24 December 2013; Published 3 February 2014

Academic Editor: Bin Zhang

Copyright (C) 2014 Suh Cem Pang et al. This is an open access article distributed under the Creative Commons Attribution License, which permits unrestricted use, distribution, and reproduction in any medium, provided the original work is properly cited.

We have demonstrated the loading of curcumin onto starch maleate (SM) under mild conditions by mixing dissolved curcumin and SM nanoparticles separately in absolute ethanol and ethanol/aqueous $(40: 60 \mathrm{v} / \mathrm{v})$, respectively. Curcumin-loaded starch-maleate (CurSM) nanoparticles were subsequently precipitated from a homogeneous mixture of these solutions in absolute ethanol based on the solvent exchange method. TEM analysis indicated that the diameters of CurSM nanoparticles were ranged between $30 \mathrm{~nm}$ and $110 \mathrm{~nm}$ with a mean diameter of $50 \mathrm{~nm}$. The curcumin loading capacity of SM as a function of loading duration was investigated using the UV-visible spectrophotometer. The loading of curcumin onto SM increased rapidly initially with loading duration, and the curcumin loading capacity of $15 \mathrm{mg} / \mathrm{g}$ was reached within 12 hours. CurSM nanoparticles exhibited substantially higher water solubility of $6.0 \times 10^{-2} \mathrm{mg} / \mathrm{mL}$ which is about 300 times higher than that of pure curcumin. With enhanced water solubility and bioaccessibility of curcumin, the potential utility of CurSM nanoparticles in various biomedical applications is therefore envisaged.

\section{Introduction}

Curcumin, a non-toxic bioactive component of turmeric even at high dosage [1], has attracted considerable attention especially for its pharmacological activities such as anticarcinogenic $[2,3]$, anti-inflammatory $[4,5]$, and antioxidant [6]. However, the utility of curcumin in clinical development and applications is limited by its low water solubility and poor bioavailability [7]. The solubility of curcumin in water is reported to be $1.99 \times 10^{-4} \mathrm{mg} / \mathrm{mL}$ [8]. However, the solubility of curcumin is reported to be $\mathrm{pH}$ dependence, and it is soluble in both strong acids [9] and dilute alkali of pH 11 [10].

Any drawback due to poor water solubility of hydrophobic bioactive agents such as curcumin could be circumvented via the development of nanoparticle-based drug delivery systems that are dispersible in aqueous media. Intense research efforts have therefore been focused on developing curcuminloaded polymeric nanoparticles for enhancing the water solubility of curcumin. Anand and co-researchers [11] have reported the synthesis of curcumin-loaded PLGA nanoparticles with enhanced water solubility. Although curcumin is pharmacologically safe for human beings, the efficacy of curcumin-loaded synthetic polymers has remained uncertain.

Various attempts have been made to synthesize polysaccharide-loaded curcumin nanoparticles. Being a type of polyphenolic molecule, curcumin could interact strongly with glucan molecule through hydrogen bonding. Such noncovalent interactions of curcumin might play a decisive role in its mechanism of actions during various pharmacological activities. Cyclodextrin, a type of polysaccharide, is known to form inclusion complexes with curcumin [12-14]. Kaminaga et al. [15] reported the conjugation of glucose molecules with curcumin molecules to form water soluble prodrugs. Gupta et al. [16] reported the encapsulation of curcumin with silk fibroin and chitosan to form curcumin-based nanoparticles using the blending method. Such encapsulation of curcumin by natural biopolymers could eliminate tissue toxicity.

In this paper, we have reported a facile synthesis approach for the preparation of water soluble curcumin-loaded starchmaleate (CurSM) nanoparticles by loading curcumin onto highly water-soluble starch-maleate monoester. The chemical structure, morphology, and mean size of CurSM nanoparticles were characterized by both FTIR and TEM. 
The curcumin loading capacity of SM nanoparticles and the resulting water solubility of CurSM nanoparticles were determined by the UV-visible spectrophotometer.

\section{Materials and Method}

2.1. Materials. Native sago starch powder was obtained from local grocery store. Sodium hydroxide (CAS No. 1310-73-2) was supplied by Mallinckrodt. Curcumin (1,7-bis(4-hydroxy3-methoxyphenyl)-1,6-heptadiene-3,5-dione) (CAS No. 45837-7) and maleic anhydride (CAS No. 108-31-6) were purchased from Merck. Absolute ethanol (CAS No. 64-17-5) was purchased from Hamburg. All chemicals were used without further purification. Ultrapure water (18.2 M $\Omega$ ) was used in all syntheses.

\subsection{Method}

2.2.1. Preparation of Curcumin-Loaded Starch-Maleate (CurSM) Nanoparticles. The preparation of starch-maleate monoester (SM) was described elsewhere with some modifications [17]. In this study, SM was prepared by dispersing $16.2 \mathrm{~g}$ native sago starch in $10 \mathrm{~mL}$ of ultrapure water and $25 \mathrm{~mL}$ of $2.0 \mathrm{M} \mathrm{NaOH}$. This was followed by adding $15.8 \mathrm{~g}$ solid maleic anhydride and heating at $80^{\circ} \mathrm{C}$ for 4 hours. SM sample was then precipitated in absolute ethanol. The loading of curcumin onto SM was carried out by mixing $1 \%$ curcumin solution in absolute ethanol with SM monoester dissolved in an ethanol/aqueous $(40: 60 \mathrm{v} / \mathrm{v})$ mixture. Typically, $0.1 \mathrm{~g}$ of SM was first dissolved in $10 \mathrm{~mL}$ of $40 \%$ ethanol, and $10 \mathrm{~mL}$ of $1 \%$ curcumin solution in absolute ethanol was added. The resulting clear yellow solution was stirred continuously at a constant stirring rate for various predetermined time intervals $(3,4,5,6,8,12$, and $16 \mathrm{hrs})$ at $50-60^{\circ} \mathrm{C}$. Absolute ethanol was then added into the SM and curcumin solution mixture. The yellow precipitate formed was rinsed several times with absolute ethanol to remove any free curcumin adhered onto surfaces of CurSM nanoparticles. Since free curcumin was completely soluble in absolute ethanol, it was removed during repeated rinsing with absolute ethanol. To ensure complete removal of free curcumin adhered onto surfaces of CurSM nanoparticles, the concentration of curcumin in the supernatant was determined by measuring the absorbance at $420 \mathrm{~nm}$ using a UV-vis spectrophotometer (Perkin Elmer/Lambda 25) against a standard curcumin solution. The yellow CurSM precipitate was dried in a conventional oven at $60^{\circ} \mathrm{C}$ for 24 hours. CurSM nanoparticles were subsequently prepared by the solvent exchange method as described previously [18]. Typically, $0.1 \mathrm{~g}$ of CurSM powder was dissolved in $10 \mathrm{~mL}$ ultrapure water and then added dropwise into $40 \mathrm{~mL}$ of absolute ethanol. The resulting nanoparticles in suspension were sonicated and centrifuged 3 times and washed with absolute ethanol to remove any free curcumin on the surface of CurSM nanoparticles. The purified CurSM nanoparticles were dried in an oven at $60^{\circ} \mathrm{C}$ for 24 hours.

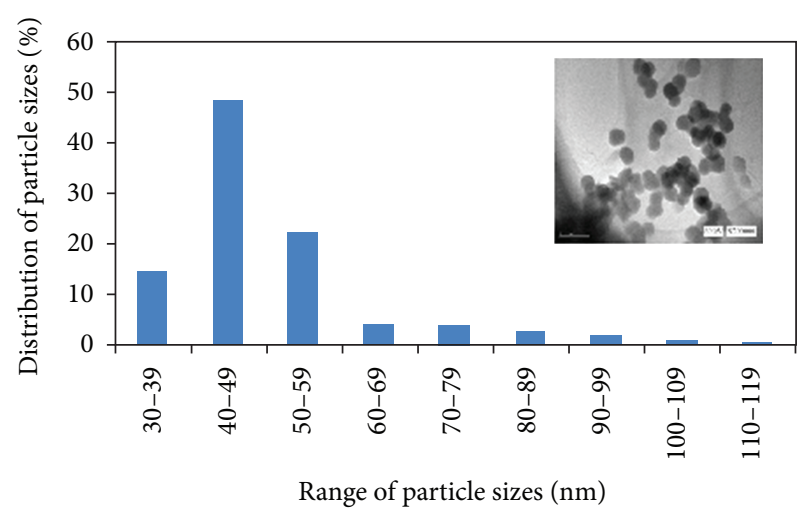

FIGURE 1: Particle size distribution of CurSM nanoparticles prepared with a loading duration of 14 hours at $50-60^{\circ} \mathrm{C}$. Inset shows the TEM micrograph of CurSM nanoparticles precipitated in absolute ethanol.

2.2.2. Curcumin Loading Capacity of Starch Maleate (SM). The total loading of curcumin onto SM was determined by dissolving CurSM nanoparticles in ultrapure water. $0.020 \mathrm{~g}$ of CurSM nanoparticles was dissolved in ultrapure water to form $5 \mathrm{~mL}$ of CurSM solution at ambient temperature. The amount of curcumin adsorbed was then determined using a UV-vis spectrophotometer (Perkin Elmer/Lambda 25) at the wavelength of $350 \mathrm{~nm}$.

2.2.3. Confocal Laser Scanning Microscope Analysis. A small drop of the CurSM dispersion in absolute ethanol was mounted on a slide and visualized using a confocal laser scanning microscope (CLSM) (LSM 410, Carl Zeiss, USA).

2.2.4. Transmission Electron Microscopy (TEM) Analysis. Dispersed CurSM samples in absolute ethanol were dropped onto formvar-coated copper grids and characterized using a transmission electron microscope (TEM) (JEOL Model JEM 1010). The mean size of CurSM nanoparticles was determined by measuring randomly 50 nanoparticles as observed in the TEM micrographs.

2.2.5. Fourier Transformed Infrared Spectrometry (FTIR) Analysis. Fourier transformed infrared spectrometry (FTIR) spectra of CurSM, SM as well as curcumin samples pelleted with potassium bromide $(\mathrm{KBr})$ were generated using a fourier transformed infrared spectrometer (SHIMADZU Model FTIR-8201PC) within the wave number range of 4000 and $400 \mathrm{~cm}^{-1}$.

\section{Results and Discussion}

3.1. Preparation of CurSM Nanoparticles. Curcumin was successfully loaded onto starch-maleate monoester (SM) and subsequently formed discrete CurSM nanoparticles of irregular distorted spherical shape via the nanoprecipitation process in absolute ethanol. Figure 1 shows the particle size distribution of CurSM nanoparticles with size ranging between 30 and $120 \mathrm{~nm}$ and a mean size of $50 \pm 12 \mathrm{~nm}$. 


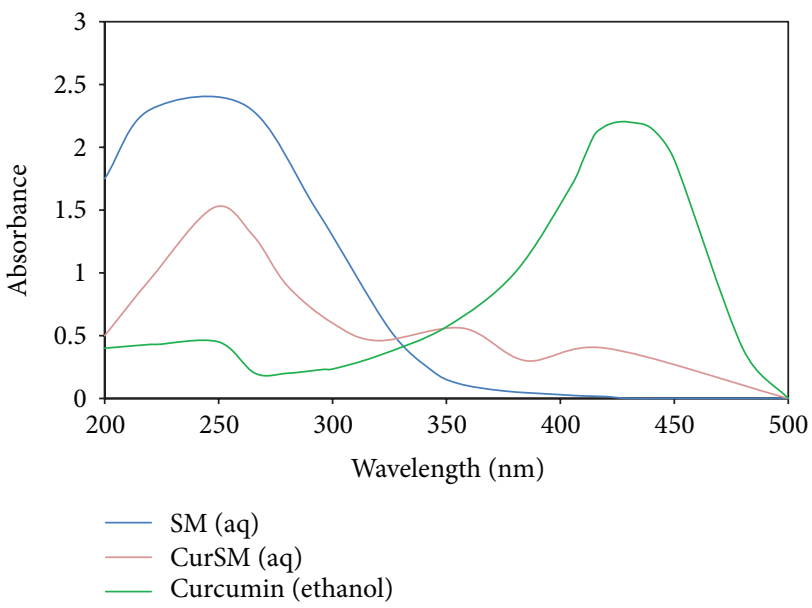

(a)

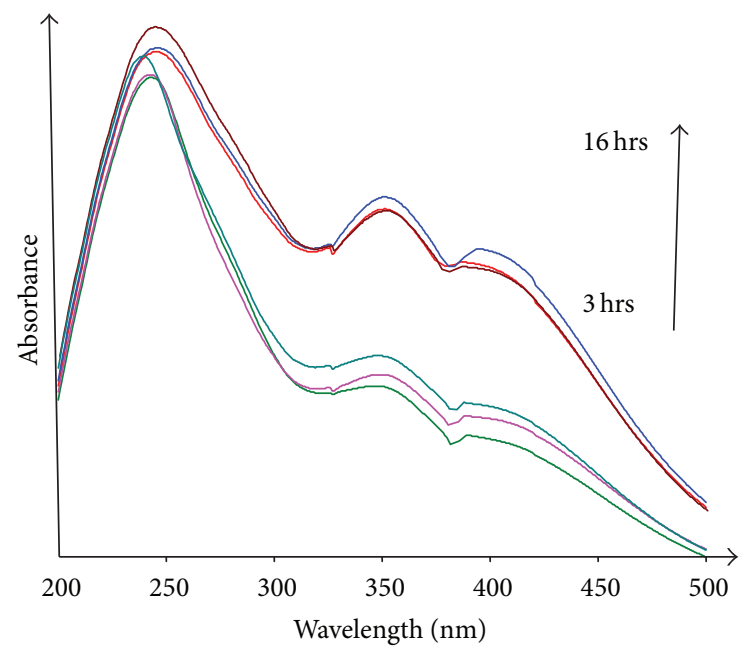

(b)

FIGURE 2: UV-visible spectra of (a) SM (aqueous), CurSM (aqueous), and curcumin (ethanolic) solutions, and (b) 0.4\% (w/v) aqueous solutions of CurSM samples prepared with different loading durations (3-16 hours).

The TEM micrograph (inset of Figure 1) shows CurSM nanoparticles of distorted spherical shape which could be attributed to their high sensitivity to gelation in the presence of water. Nevertheless, the preparation method used in the present study appeared to afford good particle size control of CurSM nanoparticles.

3.2. UV-Visible Spectra of CurSM. Figure 2(a) shows the superimposed UV-visible spectra of SM and CurSM aqueous solutions and ethanolic solution of free curcumin within the scanning wavelength range of 200 and $500 \mathrm{~nm}$. SM aqueous solution and curcumin ethanolic solution exhibited prominent absorption peaks at $250 \mathrm{~nm}$ and $420 \mathrm{~nm}$, respectively, in consonance with respective reported results $[18,19]$. The absorption peak of SM at the wavelength of $250 \mathrm{~nm}$ was attributed to the $\pi-\pi^{*}$ transition of unsaturated groups of maleate moiety within the SM molecules [18]. Free curcumin in absolute ethanol was reported to exhibit an intense, roundshaped absorption band centered at $420 \mathrm{~nm}$ in the visible region [19]. This could be attributed to its extended aromatic system with electronic dipole that allowed the $\pi-\pi^{*}$ type of excitation. Upon light absorption, a $\pi$ electron was excited from the ground state to the first excited state and oscillated from one end of the chromophore to the other. Ferrari et al. [20] reported that curcumin in physiological $\mathrm{pH}$ range undergoes tautomeric equilibrium which is highly solvent dependent. Curcumin exhibited an absorption peak in the UV-vis spectrum at around $320 \mathrm{~nm}$, which was attributed to its predominant diketo form. However, the predominant keto-enol tautomer in aprotic/apolar solvent exhibited an absorption peak at $420 \mathrm{~nm}$ [20]. Henceforth, the loading efficiency and capacity of curcumin onto starch maleate could be substantially influenced by the tautomeric equilibrium of curcumin under prevailing experimental conditions. CurSM aqueous solution showed three prominent absorption peaks at $250 \mathrm{~nm}, 350 \mathrm{~nm}$, and $420 \mathrm{~nm}$ which could be attributed to

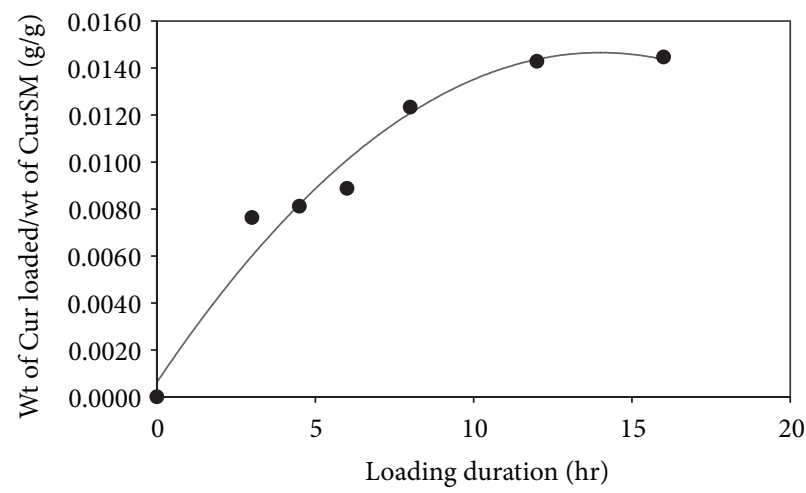

FIgURE 3: The loading profile of curcumin onto starch maleate as a function of loading duration ( $0.4 \%$ of CurSM solution).

SM, curcumin loaded within SM, and free curcumin, respectively. Intensities of these absorption peaks should correspond to their relative abundance within the CurSM sample. However, contradictory results had also been reported for free curcumin solution which exhibited a prominent peak at $350 \mathrm{~nm}$ and was attributed to the $n-\pi^{*}$ transition of the feruloyl unit of curcumin $[21,22]$.

The UV-visible spectra of CurSM aqueous solutions with samples prepared at different curcumin loading durations within the wavelength range of 200 and $400 \mathrm{~nm}$ were shown in Figure 2(b). The different intensities of two prominent peaks at wavelengths of $250 \mathrm{~nm}$ and $350 \mathrm{~nm}$ indicated the relative abundance of SM moiety and curcumin component, respectively, present in each CurSM sample. Very similar intensities of absorption peaks at $250 \mathrm{~nm}$ indicated that the concentration of SM moieties had remained almost constant throughout the curcumin loading durations. It is noteworthy that the absorption peak of curcumin within CurSM samples appeared to have blue shifted toward the near-ultraviolet $(354-366 \mathrm{~nm})$ region. Such shift could be attributed to the 


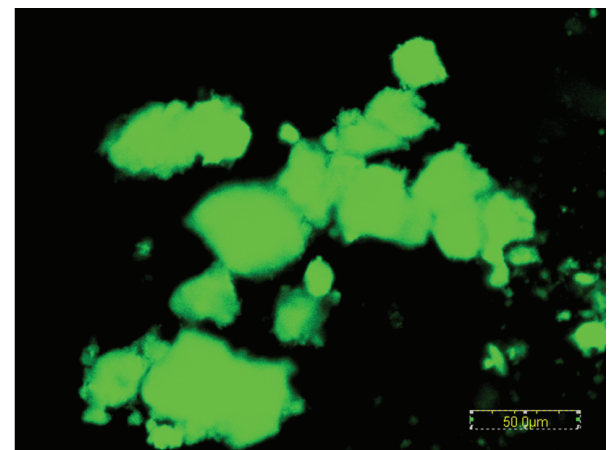

FIGURE 4: Aconfocal laser scanning micrograph of curcumin loaded starch maleate (CurSM) dispersed in absolute ethanol.

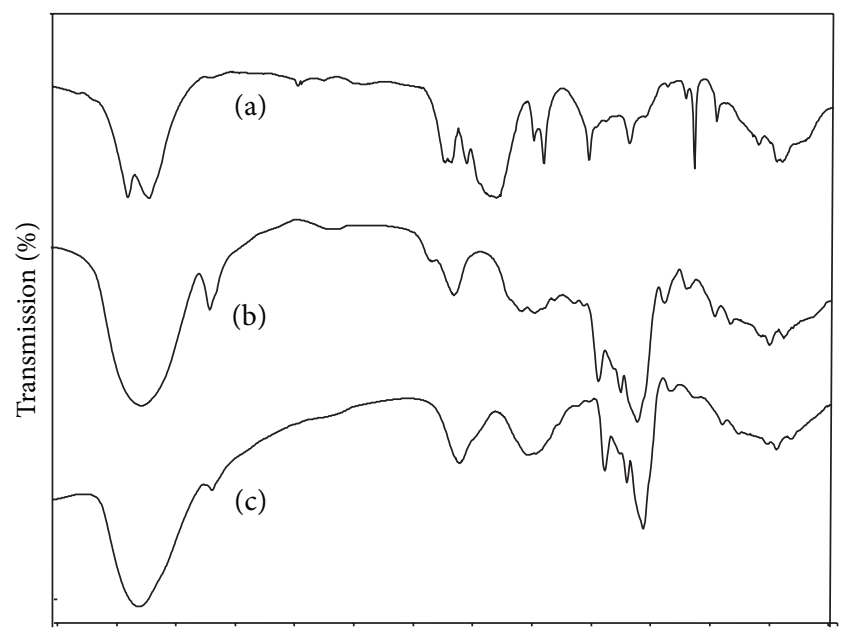

40003600320028002400200018001600140012001000800600

Wavenumber $\left(\mathrm{cm}^{-1}\right)$

FIGURE 5: FTIR spectra of (a) curcumin, (b) starch maleate (SM), and (c) curcum loaded starch-maleate (CurSM).

electronic dipole $n-\pi^{*}$ transition of the carbonyl group of curcumin, which might normally be obscured by the strong masking effect of neighboring absorption bands [6]. We therefore speculated that SM molecules in aqueous solution would interact readily with hydroxyl groups of curcumin molecules through hydrogen bonding. Such interactions would sterically prevent these molecules from adopting the planar geometry and hence gave rise to the observed shift of absorption peaks toward the near ultraviolet $(354-366 \mathrm{~nm})$ region.

Figure 3 shows the loading profile of curcumin onto SM as a function of loading duration which was monitored based on the absorbance measured at the wavelength of $350 \mathrm{~nm}$. A low temperature of $50-60^{\circ} \mathrm{C}$ was employed during the loading process in order to reduce degradation of polysaccharide chains of the SM sample. The loading of curcumin onto SM was observed to increase more rapidly initially with increasing loading durations, and the loading capacity of about $15 \mathrm{mg} / \mathrm{g}$ was reached within 12 hours.

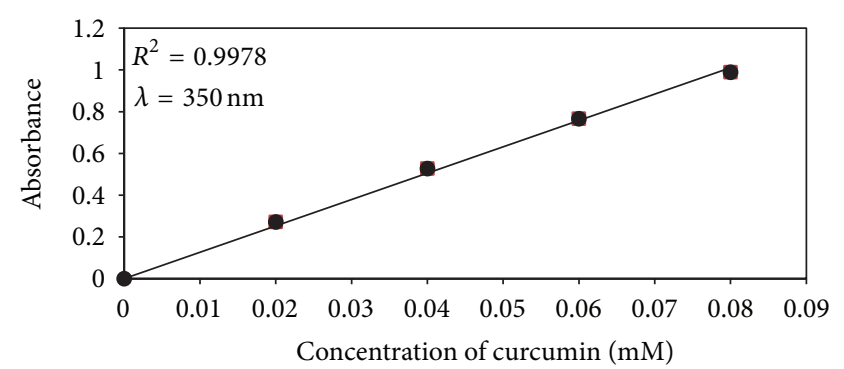

FIGURE 6: Absorbance of alkaline curcumin aqueous solution at the wavelength of $350 \mathrm{~nm}$ within the concentration range of $0-0.08 \mathrm{Mm}$.

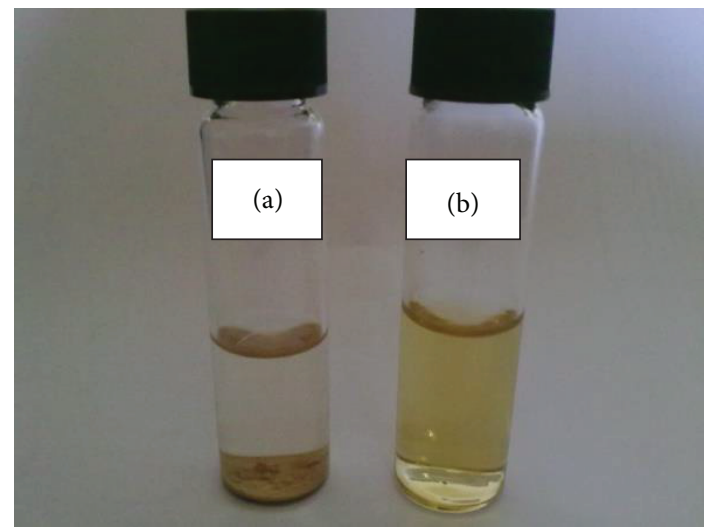

Figure 7: Photographs of CurSM samples in (a) absolute ethanol and (b) water.

Since curcumin produced natural fluorescent in the visible green spectrum, no further labeling of curcumin was necessary when it was being examined using a confocal laser scanning microscope (CLSM). Prior to CLSM analysis, the CurSM sample was rinsed three times with absolute ethanol in order to remove any free curcumin adhered onto the sample surface. As shown in Figure 4, the bright green patches and spots indicated the presence of curcumin within the CurSM sample.

3.3. FTIR Analysis. Figure 5 shows FTIR spectra of curcumin, SM, and CurSM samples. The FTIR analysis was carried out to confirm the loading of curcumin onto starchmaleate molecules. Curcumin was observed to exhibit several dominant peaks which were attributed to functional groups such as $\mathrm{O}-\mathrm{H}\left(3508 \mathrm{~cm}^{-1}\right), \mathrm{C}=\mathrm{O}, \mathrm{C}=\mathrm{C}\left(1626 \mathrm{~cm}^{-1}\right)$, and aromatic $\mathrm{C}=\mathrm{C}\left(1602 \mathrm{~cm}^{-1}\right)$, whereas $\mathrm{SM}$ showed the characteristic absorption peak at around $1700 \mathrm{~cm}^{-1}$ which was attributed to the carbonyl group. However, the characterization of CurSM samples using FTIR spectroscopy had been hindered by interfering vibrations of starch molecules at very similar wave numbers [23]. Furthermore, the conventionally high relative molecular mass of starch molecules could lead to relatively weak absorption peaks of curcumin as compared to those of starch molecules. Besides, the amorphous nature of CurSM samples had resulted in absorption peaks being less sharp and less intense than those peaks observed in the 


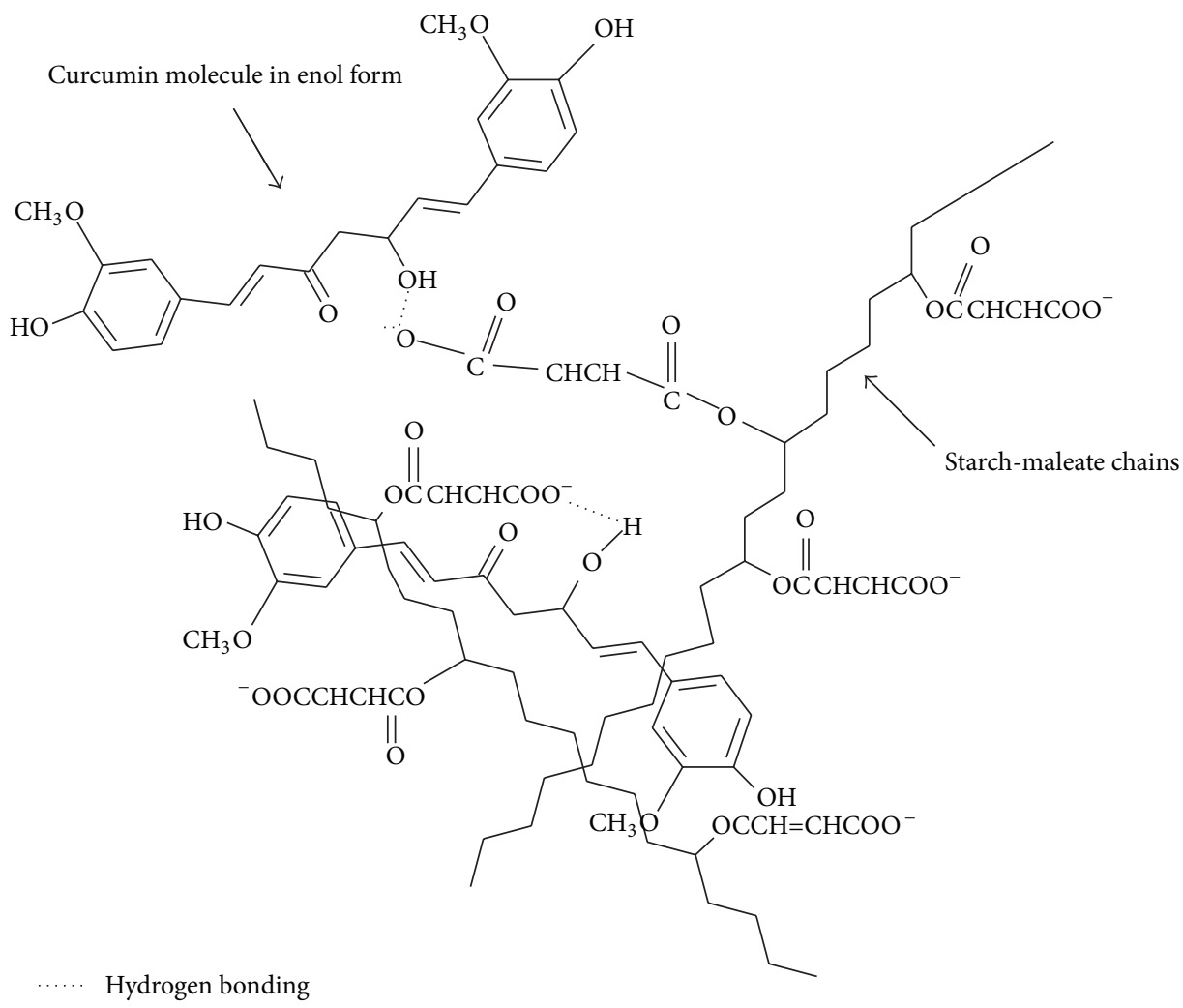

FIGURE 8: Schematic representation of interactions between curcumin molecules and starch-maleate molecules via hydrogen bonding.

spectrum of curcumin. As such, the presence of curcumin moiety within the CurSM samples could not be readily discernable in the FTIR spectra.

3.4. Solubility of Curcumin and CurSM Nanoparticles. Curcumin is extremely insoluble in water, and this feature has restricted its clinical applications. However, concerted research efforts had been focused on synthesizing water soluble curcumin derivatives due to their superior antioxidant activity against in vitro human cancers. Curcumin and curcuminoid derivatives displayed absorption peaks in the ultraviolet and visible regions. The absorbance of alkaline curcumin aqueous solution measured at the wavelength of $350 \mathrm{~nm}$ was observed to increase linearly $\left(R^{2}=0.9978\right)$ with curcumin concentration of up to $0.08 \mathrm{mM}$ at $25^{\circ} \mathrm{C}$ (Figure 6). According to some authors [24] symmetrical curcumin molecule absorbed at a wavelength of $360 \mathrm{~nm}$. A shoulder peak was observed at $360 \mathrm{~nm}$ for curcumin in basic media [25]. This showed that the solubility of curcumin in an alkaline aqueous medium could be $0.08 \mathrm{mM}$ or higher.

As shown in Figure 7, CurSM nanoparticles were visibly insoluble in absolute ethanol but readily soluble in water to produce a clear yellowish brown solution. Being a polyphenolic type of molecule, curcumin molecule was able to interact strongly with biomacromolecules through hydrogen bonding as illustrated in Figure 8. Curcumin had been shown to interact with biomacromolecules such as protein, cyclodextrin, and phospholipid [11]. As reported by several authors [14], curcumin could form water soluble complex with cyclodextrin. Hydrogen bonding interactions had been reported to occur between curcumin and other molecules such as phosphatidylcholine [26]. The aqueous solubility of CurSM nanoparticles is calculated to be $6.0 \times 10^{-2} \mathrm{mg} / \mathrm{mL}$ which is $300 \mathrm{x}$ higher than that of free curcumin $(1.99 \times$ $10^{-4} \mathrm{mg} / \mathrm{mL}$ [8]). The loading of curcumin onto water soluble starch-maleate could have resulted in substantial changes in the microenvironment of curcumin molecules due to extensive hydrogen bonding interactions between curcumin and SM molecules. Such interactions could have contributed towards enhanced solubility of curcumin in water since SM by itself was highly soluble in water.

The enhanced solubility of CurSM nanoparticles in aqueous medium could also be attributed to their nano-sized dimensions and associated large specific surface area, as well as the highly hydrophilic nature of starch-maleate molecules. Besides, traces of $\mathrm{NaOH}$ could be entrapped within CurSM samples despite our efforts to remove them by reprecipitating these CurSM samples in absolute ethanol for several times. Such traces of entrapped $\mathrm{NaOH}$ could have also contributed towards the observed higher aqueous solubility of CurSM samples.

\section{Conclusion}

The present study has demonstrated a facile approach for loading of curcumin onto starch-maleate monoester. Besides, 
water soluble curcumin-loaded starch-maleate (CurSM) nanoparticles of spherical shape were synthesized in aqueous-based system through controlled nanoprecipitation of CurSM solution in absolute ethanol. The total loading of curcumin onto SM nanoparticles could be modulated by varying the duration of loading. Due to their biocompatibility, nontoxic nature, and enhanced water solubility, the potential utility of CurSM nanoparticles in biomedical applications is therefore envisaged.

\section{Conflict of Interests}

The authors declare that there is no conflict of interests regarding the publication of this paper.

\section{Acknowledgments}

The authors wish to thank Ting Woei for her skillful assistance in carrying out the TEM works. Financial support from UNIMAS (Research Grant DI/09/2008(17)) and scholarship award from the Ministry of Education, Malaysia are gratefully acknowledged.

\section{References}

[1] M. Borsari, E. Ferrari, R. Grandi, and M. Saladini, "Curcuminoids as potential new iron-chelating agents: spectroscopic, polarographic and potentiometric study on their Fe(III) complexing ability," Inorganica Chimica Acta, vol. 328, no. 1, pp. 6168, 2002.

[2] K. K. Soudamini and R. Kuttan, "Inhibition of chemical carcinogenesis by curcumin," Journal of Ethnopharmacology, vol. 27, no. 1-2, pp. 227-233, 1989.

[3] A. Duvoix, R. Blasius, S. Delhalle et al., "Chemopreventive and therapeutic effects of curcumin," Cancer Letters, vol. 223, no. 2, pp. 181-190, 2005.

[4] M. M.-Y. Chan, H.-I. Huang, M. R. Fenton, and D. Fong, "In vivo inhibition of nitric oxide synthase gene expression by curcumin, a cancer preventive natural product with antiinflammatory properties," Biochemical Pharmacology, vol. 55, no. 12, pp. 1955-1962, 1998.

[5] K. Kohli, J. Ali, M. J. Ansari, and Z. Raheman, "Curcumin: a natural antiinflammatory agent," Indian Journal of Pharmacology, vol. 37, no. 3, pp. 141-147, 2005.

[6] F. Zsila, Z. Bikádi, and M. Simonyi, "Unique, pH-dependent biphasic band shape of the visible circular dichroism of curcumin-serum albumin complex," Biochemical and Biophysical Research Communications, vol. 301, no. 3, pp. 776-782, 2003.

[7] P. Ghandi, Z. Khan, and N. Chakraverty, "Soluble curcumin: a promising oral supplement for health management," Journal of Applied Pharmaceutical Science, vol. 1, no. 2, pp. 1-7, 2011.

[8] P. Anand, A. B. Kunnumakkara, R. A. Newman, and B. B. Aggarwal, "Bioavailability of curcumin: problems and promises," Molecular Pharmaceutics, vol. 4, no. 6, pp. 807-818, 2007.

[9] P.-H. Bong, "Spectral and photophysical behaviors of curcumin and curcuminoids," Bulletin of the Korean Chemical Society, vol. 21, no. 1, pp. 81-86, 2000.

[10] B. T. Kurien and R. H. Scofield, "Curcumin/turmeric solubilized in sodium hydroxide inhibits HNE protein modification-An in vitro study," Journal of Ethnopharmacology, vol. 110, no. 2, pp. 368-373, 2007.

[11] P. Anand, H. B. Nair, B. Sung et al., "Design of curcuminloaded PLGA nanoparticles formulation with enhanced cellular uptake, and increased bioactivity in vitro and superior bioavailability in vivo," Biochemical Pharmacology, vol. 79, no. 3, pp. 330-338, 2010.

[12] B. Tang, L. Ma, H.-Y. Wang, and G.-Y. Zhang, "Study on the supramolecular interaction of curcumin and $\alpha$-cyclodextrin by spectrophotometry and its analytical application," Journal of Agricultural and Food Chemistry, vol. 50, no. 6, pp. 1355-1361, 2002.

[13] M. A. Tomren, M. Másson, T. Loftsson, and H. H. Tønnesen, "Studies on curcumin and curcuminoids. XXXI. Symmetric and asymmetric curcuminoids: stability, activity and complexation with cyclodextrin," International Journal of Pharmaceutics, vol. 338, no. 1-2, pp. 27-34, 2007.

[14] H. H. Tønnesen, M. Másson, and T. Loftsson, "Studies of curcumin and curcuminoids. XXVII. Cyclodextrin complexation: solubility, chemical and photochemical stability," International Journal of Pharmaceutics, vol. 244, no. 1-2, pp. 127-135, 2002.

[15] Y. Kaminaga, A. Nagatsu, T. Akiyama et al., "Production of unnatural glucosides of curcumin with drastically enhanced water solubility by cell suspension cultures of Catharanthus roseus," FEBS Letters, vol. 555, no. 2, pp. 311-316, 2003.

[16] V. Gupta, A. Aseh, C. N. Ríos, B. B. Aggarwal, and A. B. Mathur, "Fabrication and characterization of silk fibroin-derived curcumin nanoparticles for cancer therapy," International Journal of Nanomedicine, vol. 4, pp. 115-122, 2009.

[17] S. C. Pang, S. F. Chin, S. H. Tay, and F. M. Tchong, "Starchmaleate-polyvinyl alcohol hydrogels with controllable swelling behaviors," Carbohydrate Polymers, vol. 84, no. 1, pp. 424-429, 2011.

[18] S. H. Tay, S. C. Pang, and S. F. Chin, "Facile synthesis of starchmaleate monoesters from native sago starch," Carbohydrate Polymers, vol. 88, no. 4, pp. 1195-1200, 2012.

[19] P. R. Krishna Mohan, G. Sreelakshmi, C. V. Muraleedharan, and R. Joseph, "Water soluble complexes of curcumin with cyclodextrins: characterization by FT-Raman Spectroscopy," Vibrational Spectroscopy, vol. 62, pp. 77-84, 2012.

[20] E. Ferrari, F. Pignedoli, C. Imbriano et al., "Newly synthesized curcumin derivatives: crosstalk between chemicophysical properties and biological activity," Journal of Medicinal Chemistry, vol. 54, no. 23, pp. 8066-8077, 2011.

[21] X. Wang, Y. Jiang, Y.-W. Wang, M.-T. Huang, C.-T. Ho, and Q. Huang, "Enhancing anti-inflammation activity of curcumin through O/W nanoemulsions," Food Chemistry, vol. 108, no. 2, pp. 419-424, 2008.

[22] M. Bernabé-Pineda, M. T. Ramírez-Silva, M. A. Romero-Romo, E. González-Vergara, and A. Rojas-Hernández, "Spectrophotometric and electrochemical determination of the formation constants of the complexes Curcumin-Fe(III)-water and Curcumin-Fe(II)-water," Spectrochimica Acta A, vol. 60, no. 5, pp. 1105-1113, 2004.

[23] G. Began, E. Sudharshan, K. Udaya Sanka, and A. G. Appu Rao, "Interaction of curcumin with phosphatidylcholine: a spectrofluorometric study," Journal of Agricultural and Food Chemistry, vol. 47, no. 12, pp. 4992-4997, 1999.

[24] S. F. Chin, K. S. Iyer, M. Saunders et al., "Encapsulation and sustained release of curcumin using superparamagnetic silica reservoirs," Chemistry, vol. 15, no. 23, pp. 5661-5665, 2009. 
[25] A. Barik, B. Mishra, A. Kunwar et al., "Comparative study of copper(II)-curcumin complexes as superoxide dismutase mimics and free radical scavengers," European Journal of Medicinal Chemistry, vol. 42, no. 4, pp. 431-439, 2007.

[26] D. Ke, X. Wang, Q. Yang et al., "Spectrometric study on the interaction of dodecyltrimethylammonium bromide with curcumin," Langmuir, vol. 27, no. 23, pp. 14112-14117, 2011. 

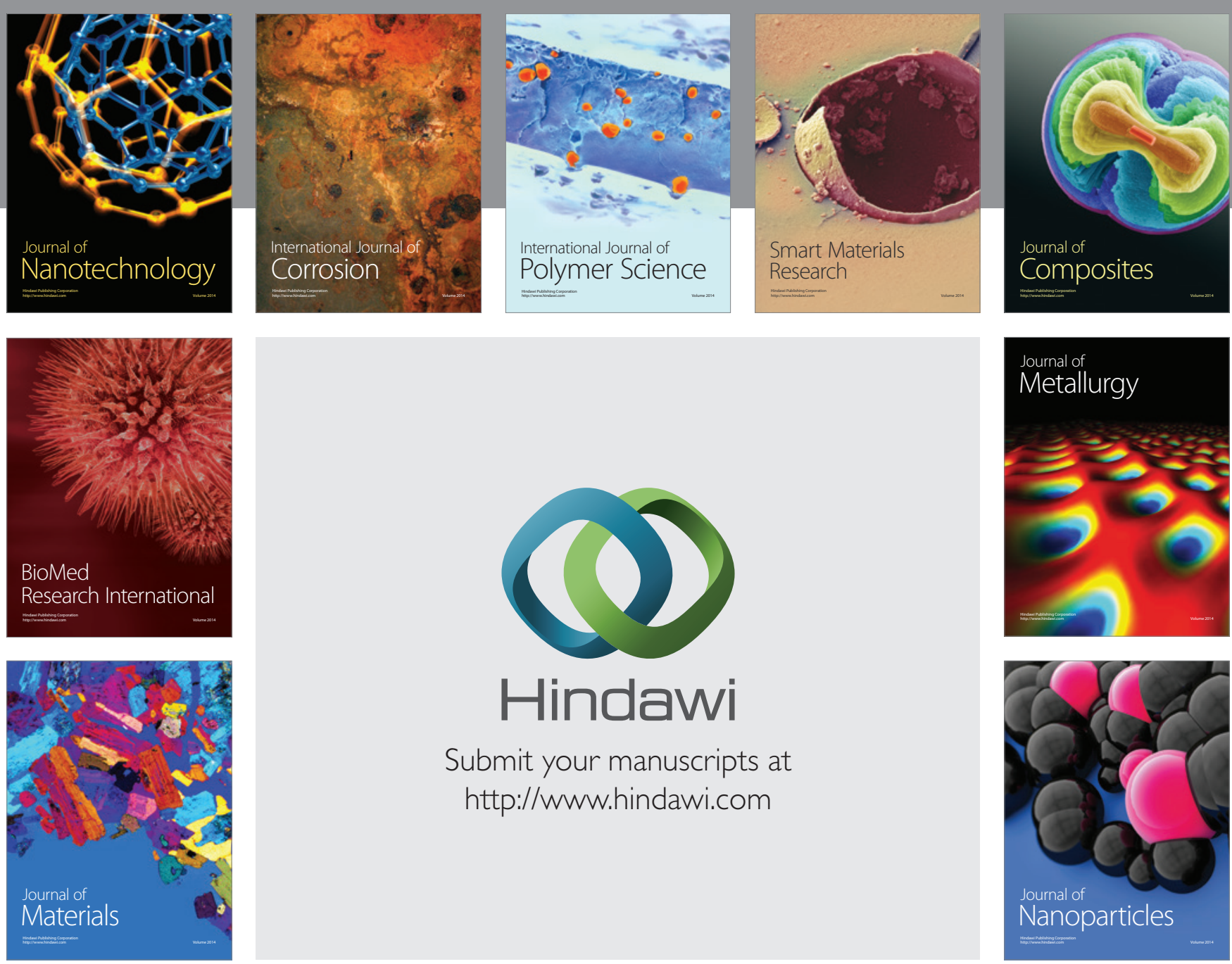

Submit your manuscripts at http://www.hindawi.com
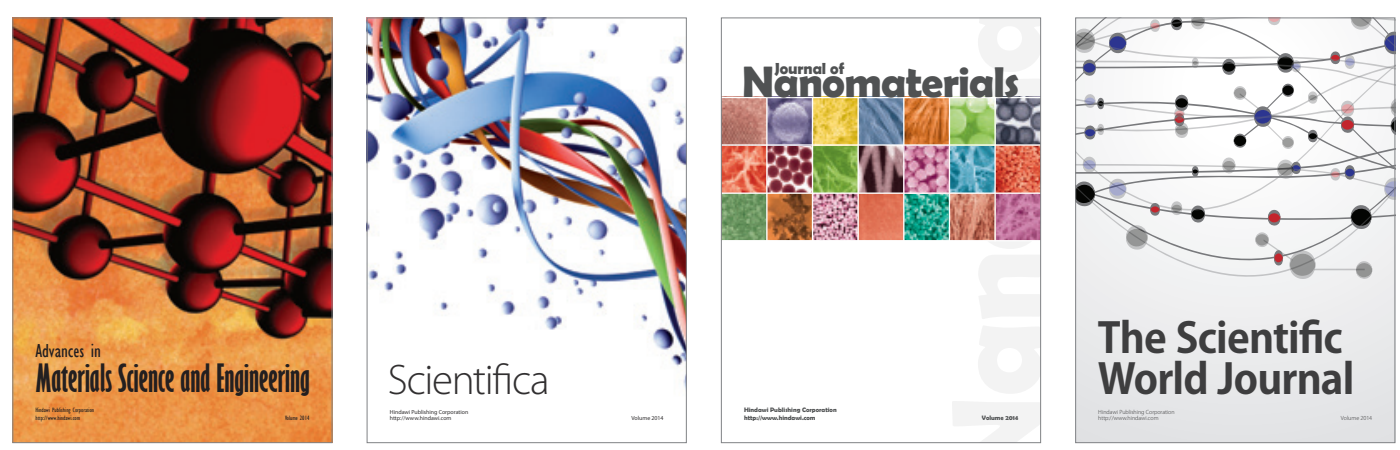

\section{The Scientific World Journal}
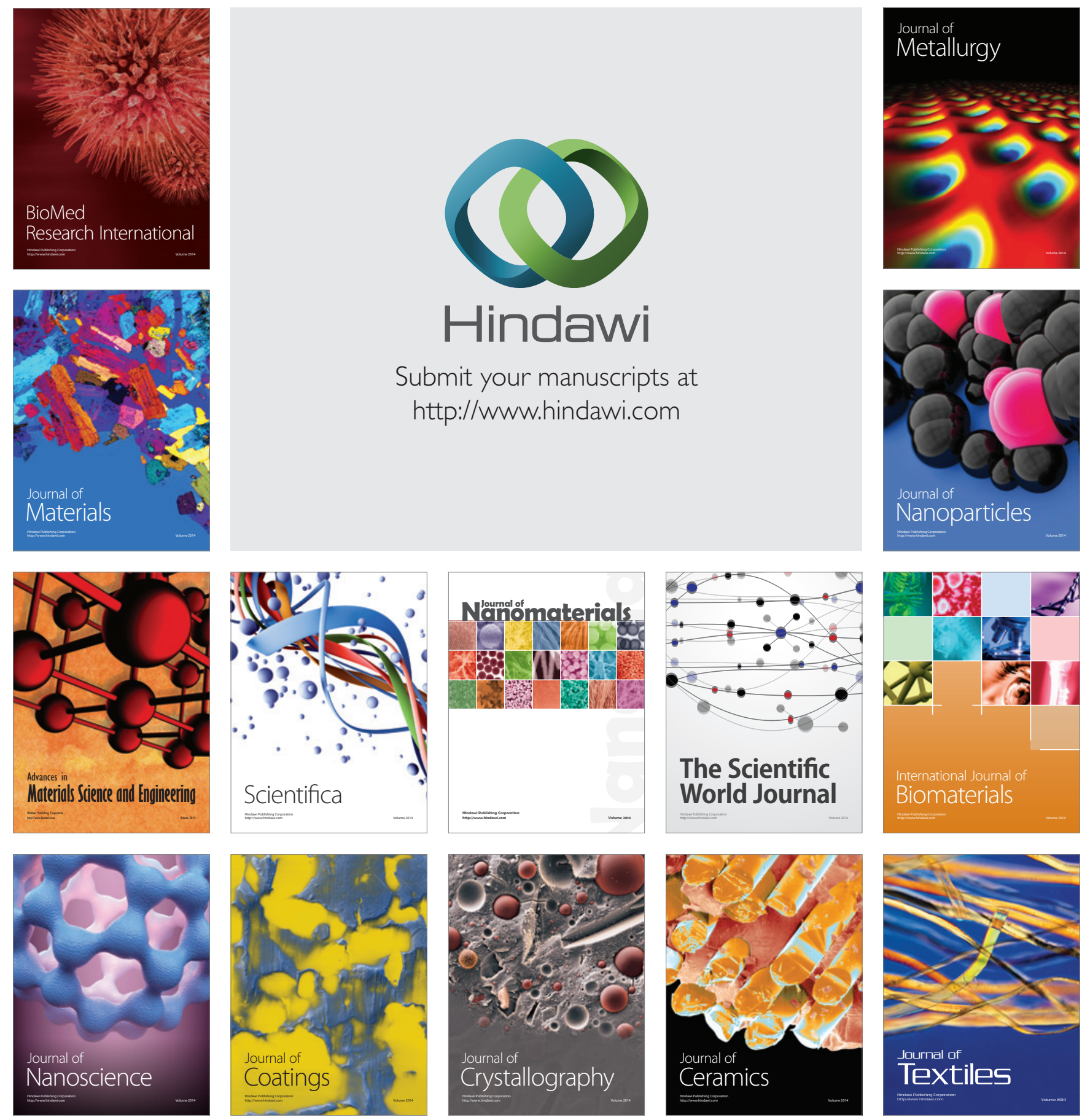